\title{
Relaxin ameliorates high glucose-induced cardiomyocyte hypertrophy and apoptosis via the Notch1 pathway
}

\author{
XIAO WEI $^{1}$, YUAN YANG ${ }^{1}$, YIN-JIU JIANG ${ }^{2}$, JIAN-MING LEI ${ }^{1}$, JING-WEN GUO $^{1}$ and HUA XIAO ${ }^{1}$ \\ Departments of ${ }^{1}$ Cardiology and ${ }^{2}$ Thoracic Surgery, \\ The First Affiliated Hospital of Chongqing Medical University, Chongqing 400016, P.R. China
}

Received April 11, 2017; Accepted August 21, 2017

DOI: $10.3892 /$ etm.2017.5448

\begin{abstract}
The present study aimed to investigate the role of relaxin (RLX) on high glucose (HG)-induced cardiomyocyte hypertrophy and apoptosis, as well as the possible molecular mechanism. H9c2 cells were exposed to $33 \mathrm{mmol} / \mathrm{l} \mathrm{HG}$ with or without RLX (100 nmol/ml). Cell viability, apoptosis, oxidative stress, cell hypertrophy and the levels of Notch1, hairy and enhancer of split 1 (hes1), atrial natriuretic polypeptide (ANP), brain natriuretic peptide (BNP), manganese superoxide dismutase (MnSOD), cytochrome $\mathrm{C}$ and caspase-3 were assessed in cardiomyocytes. Compared with the HG group, the viability of H9c2 cells was increased by RLX in a time- and dose-dependent manner, and was accompanied with a significant reduction in apoptosis. Furthermore, RLX significantly suppressed the formation of reactive oxygen species and malondialdehyde, and enhanced the activity of SOD. In addition, the levels of ANP, BNP, cytochrome C and caspase-3 were increased and Notch1, hes1 and MnSOD were inhibited in the HG group compared with those in the normal group. However, the Notch inhibitor DAPT almost abolished the protective effects of RLX. These results suggested that RLX protected cardiomyocytes from HG-induced hypertrophy and apoptosis partly through a Notch1-dependent pathway, which may be associated with reducing oxidative stress.
\end{abstract}

\section{Introduction}

Diabetic cardiomyopathy (DCM), a common complication of diabetes, is a major cause of morbidity and mortality among

Correspondence to: Dr Hua Xiao, Department of Cardiology, The First Affiliated Hospital of Chongqing Medical University, 1 Youyi Road, Chongqing 400016, P.R. China

E-mail: xiaoheart1974@163.com

Abbreviations: RLX, relaxin; HG, high glucose; ANP, atrial natriuretic polypeptide; BNP, brain natriuretic peptide; ROS, reactive oxygen species; MDA, malondialdehyde; SOD, superoxide dismutase

Key words: apoptosis, diabetic cardiomyopathy, hypertrophy, notch pathway, oxidative stress, relaxin diabetic patients (1). Rubler et al (2) was the first to introduce the term 'diabetic cardiomyopathy'. DCM is characterized by a set of impairments, including oxidative stress, cardiomyocyte hypertrophy, myocardial apoptosis and fibrosis, impaired diastolic function and eventually cardiac failure (3). High glucose (HG)-mediated oxidative stress has been considered as a major causal risk factor that triggers hypertrophic and apoptotic responses, which ultimately contributes to the pathogenesis of DCM $(4,5)$. Therefore, scavenging of reactive oxygen species (ROS) and reducing cardiomyocyte hypertrophy and apoptosis may effectively prevent the initiation or progression of DCM.

Relaxin (RLX), a pleiotropic hormone, was first identified for its role in the reproduction and pregnancy (6), but is now best known for its vasodilation effects, antiangiogenic and antifibrotic properties, as well as cardioprotection in various experimental models $(7,8)$. Previous studies have indicated that atrial cardiomyocytes produce RLX (9) and that the RLX family peptide receptor-1 (RXFP1) is expressed by atrial ventricular cells (10) and H9C2 cardiomyocytes (11). In addition, the density of RXFP1 in the heart was higher than that in uterine myometrium, indicating that RLX has an important role in cardiovascular tissues (12). It has been demonstrated that $\mathrm{HG}$ promotes the expression of endogenous RLX and that RLX inhibited cardiac fibrosis under HG conditions (13). Furthermore, RLX protected cardiomyocytes from HG-mediated apoptosis by suppressing apoptosis-associated pathways and endoplasmic reticulum stress (14). RLX robustly inhibits cardiomyocyte hypertrophy in vivo (15) and in vitro (16). These studies suggested the beneficial effects of RLX on cardiovascular complications.

Notch1 signaling is mainly involved in the important processes of cell fate determination, proliferation, differentiation and regeneration (17). Studies have indicated that the activation of Notch1 signaling protects against cardiac myocyte injury, while inhibition of the Notch1 pathway exacerbates myocardial hypertrophy, apoptosis (18) and fibrosis (19), and may eventually lead to dilated myopathy (20). An increasing number of studies have supported the association between RLX and Notch1 by demonstrating that RLX prevents cardiac fibroblast to myofibroblast and endothelial to mesenchymal transition involving the Notch1 pathway $(21,22)$. Boccalini et al (11) also concluded that RLX-activated Notch1 signaling protects cardiac myocyte cells from 
hypoxia/reoxygenation injury. However, it has remained elusive whether RLX ameliorates hyperglycemia-induced cardiomyocyte hypertrophy and apoptosis via activation of the Notch1 pathway. Hence, the present study was designed to explore the potential protective mechanisms of RLX on $\mathrm{H} 9 \mathrm{c} 2$ cells using an in vitro model of hyperglycemia-induced myocardial injury. The present study may provide possible molecular mechanisms underlying the effect of RLX as a potent treatment for DCM.

\section{Materials and methods}

Cell culture and treatments. Embryonic rat cardiac $\mathrm{H} 9 \mathrm{c} 2$ cells (Chinese Academy of Sciences, Shanghai, China) were cultured in Dulbecco's modified Eagle's medium (HyClone; GE Healthcare, Little Chalfont, UK) supplemented with $100 \mathrm{U} / \mathrm{ml}$ penicillin and streptomycin, and $10 \%$ fetal bovine serum (PAN Biotech, Aidenbach, Germany) at $37^{\circ} \mathrm{C}$ in a humidified atmosphere containing $5 \% \mathrm{CO}_{2}$. $\mathrm{H} 9 \mathrm{c} 2$ cells have numerous similarities with primary cardiomyocytes and are commonly used as a model of hypertrophy or apoptosis. In addition, compared with H9c2 cells, cardiomyocyte hypertrophy and apoptosis in primary neonatal heart cells are difficult to discern due to varying shapes and cell clumping. Therefore, the present study used the H9c2 cell line. Cells between passages 3 and 5 were used for each experiment. H9c2 cells were randomly divided into four groups for treatment: Normal control group (NG, $5.5 \mathrm{mmol} / \mathrm{l})$; high glucose group ( $\mathrm{HG}, 33 \mathrm{mmol} / \mathrm{l})$; high glucose + relaxin group (HG + RLX group); high glucose + relaxin $+\mathrm{N}$-(N-(3,5-difluorophenacetyl)-L-alanyl)-S-phenylglycine t-butyl ester (DAPT) group ( $\mathrm{HG}+\mathrm{RLX}+\mathrm{DAPT}$ group). Cells in the logarithmic growth phase were cultured in normal glucose medium for $24 \mathrm{~h}$, and then stimulated with $\mathrm{HG}$ medium with or without RLX (100 nmol/ml; cat. no. 130-15; PeproTech, Inc., Rocky Hill, NJ, USA) for 48 h. The Notch1 inhibitor DAPT (10 $\mu \mathrm{mol} / 1$; cat. no. D5942; Sigma-Aldrich; Merck KGaA, Darmstadt, Germany) was administered $4 \mathrm{~h}$ prior to HG exposure and subsequent stimulation with RLX for $48 \mathrm{~h}$. The cells were then harvested and used in the following experiments.

Cell counting Kit-8 (CCK-8) viability assay. The viability of cells was measured using the CCK-8 assay (cat. no. CK04; Dojindo, Kumamoto, Japan) according to the manufacturer's protocol. In brief, cells were seeded onto 96-well plates overnight $\left(1 \times 10^{3}\right.$ cells per well) and subsequently treated with different concentrations of $\operatorname{RLX}(0,25,50,100$ or $200 \mathrm{nmol} / \mathrm{ml})$ and for different durations (24, 48 or $72 \mathrm{~h}$ ). Subsequently, cells were incubated with $10 \mu \mathrm{mol}$ CCK- 8 solution under normal incubation conditions for $2 \mathrm{~h}$. The absorbance at $450 \mathrm{~nm}$, as an indicator of cell viability, was measured using a microplate reader (Multiskan MK3; Thermo Fisher Scientific Inc., Waltham, MA, USA).

Cell surface area measurement. The surface area of cardiac myocytes, as a hallmark of hypertrophy, was quantified using tetramethylrhodamine B isothiocyanate-labeled phalloidin (cat. no. P1951; Sigma-Aldrich; Merck KGaA). In brief, cells were grown on ordinary coverslips $(18 \times 18 \mathrm{~mm})$ in 24 -well plates $\left(1 \times 10^{4}\right.$ cells per well) to either $90 \%$ confluence or subconfluence, and then treated as previously described. H9c2 cells were then fixed with $4 \%$ paraformaldehyde and blocked with 10\% goat serum (cat. no. AR1009; Boster Biological Technology, Pleasanton, CA, USA) for $1 \mathrm{~h}$ at room temperture. After washing with PBS three times, the cells were stained using tetramethylrhodamine B isothiocyanate-labeled phalloidin and incubated for $30 \mathrm{~min}$ at room temperature in the dark, and then counterstained with DAPI (cat. no. C1006; Beyotime Institute of Biotechnology, Shanghai, China) and imaged with a fluorescence microscope (FLX 800; Olympus, Tokyo, Japan). Finally, the cell size was analyzed using Image-Pro Plus image analysis software (version 6.0; Media Cybernetics, Inc., Rockville, MD, USA).

Cell apoptosis assay. Cell apoptosis was analyzed using a Annexin V-fluorescein isothiocyanate (FITC) and propidium iodide (PI) double staining apoptosis detection kit (cat. no. G003; Nanjing Jiancheng Bioengineering Institute, Nanjing, China) and flow cytometry. In brief, after incubation as previously described, the H9c2 cells were collected, resuspended in binding buffer and then stained with Annexin V-FITC and PI solution for $15 \mathrm{~min}$ at $37^{\circ} \mathrm{C}$ in the dark. Finally, cell samples were quantified by flow cytometric analysis (BD FACSVantage $^{\mathrm{TM}}$ SE; BD Biosciences, Franklin Lakes, NJ, USA). The index of apoptosis was expressed as the percentage of total apoptotic cells, which included the percentage of early apoptotic cells (Annexin V-positive and PI-negative) plus the percentage of late apoptotic cells (Annexin V-positive and PI-positive).

Intracellular ROS measurement. Intracellular ROS was assessed using the fluorescent probe 2',7'-dichlorofluorescin diacetate (DCFH-DA) (cat. no. S0033; Beyotime Institute of Biotechnology). In brief, after incubation as mentioned above, the H9c2 cells were stained with $10 \mu \mathrm{mol}$ DCFH-DA in PBS for $30 \mathrm{~min}$ at $37^{\circ} \mathrm{C}$ in the dark. The fluorescence intensity was determined by flow cytometry and in addition, images were captured with a fluorescence microscope (FLX 800; Olympus) with an excitation and emission wavelength of 488 and $525 \mathrm{~nm}$, respectively. The mean fluorescence intensity was calculated using Image-Pro Plus software (version 6.0).

Malondialdehyde (MDA) and superoxide dismutase (SOD) measurements. The activity of SOD (cat. no. A001-3) and the levels of MDA (cat. no. A003-1) (both Nanjing Jiancheng Bioengineering Institute) were detected with the relevant detection kits according to the manufacturer's protocol. In brief, after incubation as described above, the H9c2 cells were harvested, lysates were prepared by ultrasonication at 24 $\mathrm{KHz}$ amplitude for $2 \mathrm{sec}$, all steps were performed on ice and repeated five times, and further centrifuged at 12,000 $\mathrm{x} \mathrm{g}$ for $10 \mathrm{~min}$ at $4^{\circ} \mathrm{C}$. The absorbance of the colorimetric stains was determined at wavelengths of 532 and $450 \mathrm{~nm}$, respectively. MDA and SOD levels were calculated using standard curves and normalized to the normal control.

Reverse-transcription quantitative polymerase chain reaction $(R T-q P C R)$. Total RNA was extracted from cells using RNAiso Plus reagent (cat. no. 9108; Takara Bio Inc., Dalian, China) and complementary DNA was synthesized using a PrimeScript ${ }^{\mathrm{TM}}$ 
RT reagent kit (Perfect Real Time) (cat. no. RR037A; Takara Bio Inc.). PCR amplification reactions were performed on an ABI-PRISM 7700 Sequence Detection system (Applied Biosystems; Thermo Fisher Scientific, Inc.) with the SYBR ${ }^{\circledR}$ Premix Ex Taq ${ }^{\mathrm{TM}}$ II (Tli RNase H Plus) (cat. no. RR820A; Takara Bio Inc.) and the primers listed in Table I. The PCR amplification conditions were as follows: $95^{\circ} \mathrm{C}$ for $30 \mathrm{sec}$, followed by 40 cycles of $95^{\circ} \mathrm{C}$ for $5 \mathrm{sec}$ and $60^{\circ} \mathrm{C}$ for $30 \mathrm{sec}$ for denaturation, annealing and elongation. The PCR products were quantified using the $2^{-\Delta \Delta C q}$ comparative method (23) with GAPDH as an internal control.

Western blot analysis. Cells were lysed with radioimmunoprecipitation assay buffer supplemented with a mixture of protease inhibitors (Beyotime Institute of Biotechnology), and the lysates were clarified by centrifugation at $12,000 \mathrm{x} g$ for $15 \mathrm{~min}$ at $4^{\circ} \mathrm{C}$. Protein concentrations were quantified using a bicinchoninic acid assay kit (Thermo Fisher Scientific, Inc.). Equal amounts of protein $(10 \mu \mathrm{g} /$ lane $)$ were subjected to $12 \%$ SDS-PAGE and electrotransferred onto polyvinylidene fluoride membranes (EMD Millipore, Billerica, MA, USA). Subsequently, the membranes were blocked with $5 \%$ skimmed milk for $2 \mathrm{~h}$ at room temperature and incubated overnight at $4^{\circ} \mathrm{C}$ with primary antibodies to Notch 1 (cat. no. ab52301), which recognized Notch-1 receptor and its activated form Notch-ICD, atrial natriuretic polypeptide (ANP; cat. no. ab189921), hairy and enhancer of split 1 (hes1; cat. no. ab108937) (all 1:1,000; Abcam, Cambridge, MA, USA), brain natriuretic peptide (BNP; cat. no. w102126), MnSOD (cat. no. w102506;), cytochrome C (cat. no. w101571), caspase-3 (cat. no. wl01992) (all 1:500; Wanlei Biotechnology, Shenyang, China) and GAPDH (1:1,000; cat. no. 5174; Cell Signaling Technology, Inc., Danvers, MA, USA). Finally, the membranes were incubated with horseradish peroxidase-conjugated goat anti-rabbit immunoglobulin G (1:5,000; cat. no. BA1054; Boster Biological Technology) for $2 \mathrm{~h}$ at room temperature. Proteins were detected using enhanced chemiluminescent reagent (Beyotime Institute of Biotechnology) and images were captured with a ChemiDoc XRS detection system (Bio-Rad Laboratories, Inc., Hercules, CA, USA). The values were normalized to GAPDH and densitometric analysis of the bands was performed using Fusion-Capt analysis software (Vilber Lourmat Deutschland GmbH, Eberhardzell, Germany).

Statistical analysis. Statistical analysis was performed using SPSS 19.0 software (IBM Corp., Armonk, NY, USA). Values are expressed as the mean \pm standard deviation. Comparisons between groups were made using one-way analysis of variance followed by the Tukey's t-test. $\mathrm{P}<0.05$ was considered to indicate a statistically significant difference.

\section{Results}

$R L X$ increases $H G$-induced cell viability and decreases apoptosis. The cell viability in the normal control group was defined as $100 \%$, while that in the other groups was presented as the percentage of the control group. The CCK8 assay revealed that $\mathrm{HG}$ caused a marked reduction in the viability of the H9c2 cells when compared with that in the control group $(\mathrm{P}<0.01$; Fig. 1A). However, the viability of $\mathrm{H} 9 \mathrm{c} 2$ cells
Table I. Primers sequences used for reverse-transcription quantitative polymerase chain reaction analysis.

\begin{tabular}{|c|c|}
\hline Gene & Primers $5^{\prime}-3^{\prime}$ \\
\hline Notch1 & $\begin{array}{l}\text { Forward: CCTTGTCCCCGATTATCTACCA } \\
\text { Reverse: CAGGTTCTGAGGCT GGATTTGT }\end{array}$ \\
\hline hes 1 & $\begin{array}{l}\text { Forward: GGCCAATTTGCTTTCCTCATC } \\
\text { Reverse: GAAGGCGACACTGCGTTAGG }\end{array}$ \\
\hline ANP & $\begin{array}{l}\text { Forward: CTGGGGAAGTCAACCCGTCT } \\
\text { Reverse: TCTGGGCTCCAATCCTGTCA }\end{array}$ \\
\hline BNP & $\begin{array}{l}\text { Forward: AGCCAGTCTCCAGAACAATCCA } \\
\text { Reverse: TGTGCCATCTTGGAATTTCGA }\end{array}$ \\
\hline GAPDH & $\begin{array}{l}\text { Forward: GACATGCCGCCTGGAGAAC } \\
\text { Reverse: AGCCCAGGATGCCCTTTAGT }\end{array}$ \\
\hline
\end{tabular}

ANP, atrial natriuretic polypeptide; hes1, hairy and enhancer of split 1; BNP, brain natriuretic peptide.

was statistically increased by RLX in the $50 \mathrm{nmol} / \mathrm{ml}$ group $(\mathrm{P}<0.05)$. With a further increase of the RLX concentration, the viability of cells was considerably increased by RLX in the 100 and $200 \mathrm{nmol} / \mathrm{ml}$ groups $(\mathrm{P}<0.01)$. In addition, with the prolongation of the incubation time, the viability of $\mathrm{H} 9 \mathrm{c} 2$ cells was gradually increased by RLX and significantly increased after $48 \mathrm{~h}(\mathrm{P}<0.01)$ (data not shown). These results indicated that RLX increased the cell viability in a dose- and time-dependent manner. Accordingly, $100 \mathrm{nmol} / \mathrm{ml} \mathrm{RLX}$ was applied to $\mathrm{H} 9 \mathrm{c} 2$ cardiomyocytes for a duration of $48 \mathrm{~h}$ in the following experiments.

To test the effect of RLX on cardiac apoptosis in H9c2 cells exposed to HG, Annexin V-FITC/PI dual staining and flow cytometry were used. As presented in Fig. 1B and C. the early and late apoptotic rates were significantly increased in the HG group compared with those in the control group $(\mathrm{P}<0.01)$. When compared with the HG group, addition of RLX resulted in a noticeable decrease in apoptotic cells $(\mathrm{P}<0.01)$, which was consistent with the results of previous studies $(14,16)$. Furthermore, H9c2 cells were incubated with the Notch inhibitor DAPT for $4 \mathrm{~h}$ prior to treatment with HG. The number of apoptotic cells was increased when compared with that in the HG + RLX group $(\mathrm{P}<0.01)$.

RLX attenuates $H G$-induced cardiomyocyte hypertrophy. To assess cellular hypertrophy, the cell surface area was determined by fluorescence microscopy. As displayed in Fig. 2A and $\mathrm{B}, \mathrm{H} 9 \mathrm{c} 2$ cells treated with $\mathrm{HG}$ for $48 \mathrm{~h}$ demonstrated a marked increase in cell size compared with that in the control group $(\mathrm{P}<0.01)$, which was reduced by simultaneous treatment with RLX $(\mathrm{P}<0.05)$. However, pretreatment with DAPT markedly enhanced the cell surface area compared with that in the HG+RLX group $(\mathrm{P}<0.05)$.

$R L X$ prevents $H G$-induced oxidative stress. To determine whether the HG-mediated increase in oxidative stress is critical for the effects of RLX on myocardial injury, the intracellular levels of oxidative stress were determined by DCFH-DA 
A

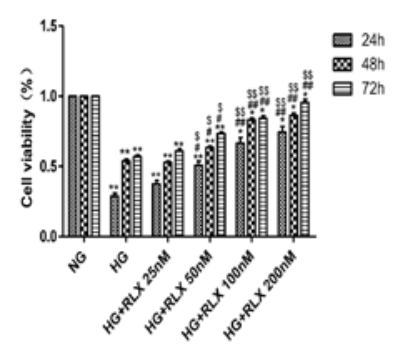

C

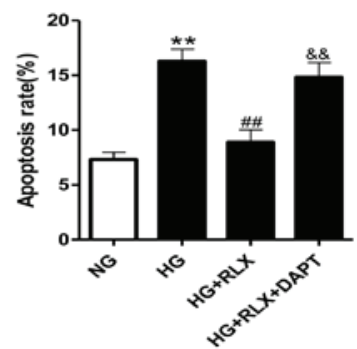

B
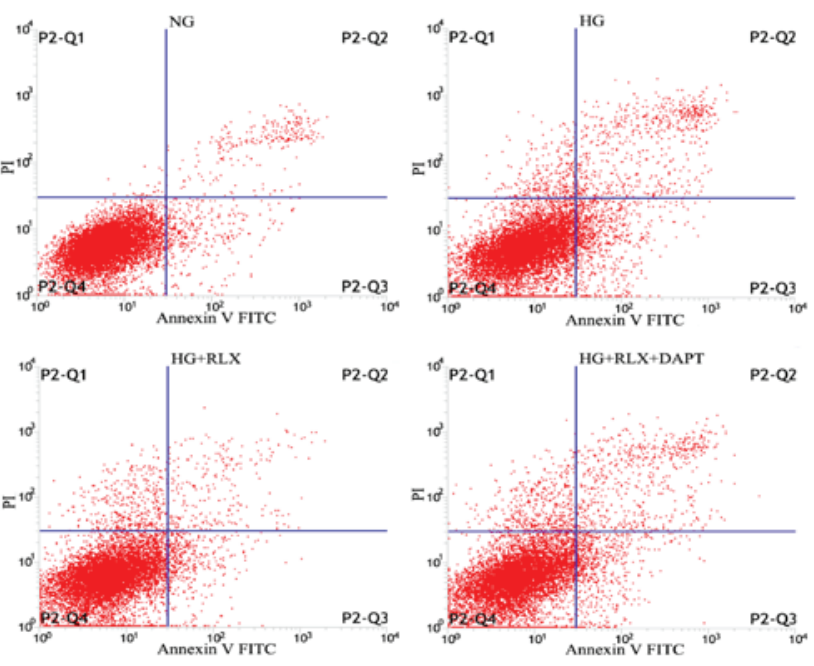

Figure 1. RLX increases cell viability in a dose- and time-dependent manner and reduces apoptosis of $\mathrm{H} 9 \mathrm{c} 2$ cells exposed to HG. The cell viability in the normal control group was defined as $100 \%$, while that in the other groups was presented as the percentage of the control group. (A) Cell viability was measured by a Cell Counting Kit- 8 assay. (B) Cell apoptosis was measured using Annexin-V/PI staining and flow cytometry. P2-Q1 represents the percentages of dead cells, $\mathrm{P} 2-\mathrm{Q} 2$ represents the percentages of cells in early apoptosis, $\mathrm{P} 2-\mathrm{Q} 3$ represents the percentages of cells in late apoptosis and P2-Q4 represents the percentages of viable cells. (C) Quantitative analysis of flow cytometry results. Values are expressed as the mean \pm standard deviation ( $\mathrm{n}=6$ in each group). ${ }^{*} \mathrm{P}<0.05$ and ${ }^{* *} \mathrm{P}<0.01$ vs. NG group; ${ }^{*} \mathrm{P}<0.05$ and ${ }^{\# \#} \mathrm{P}<0.01$ vs. HG group; ${ }^{\$} \mathrm{P}<0.05$ and ${ }^{\$ \$} \mathrm{P}<0.01$ vs. $\mathrm{HG}+\mathrm{RLX}(25 \mathrm{nM})$ group; \&\& $\mathrm{P}<0.01$ vs. HG + RLX group. FITC, fluorescein isothiocyanate; $\mathrm{P} 2$, the number of cells; PI, propidium iodide; $\mathrm{Q}$, quadrant; $\mathrm{NG}$, normal glucose; $\mathrm{HG}$, high glucose; RLX, relaxin.

staining. It was demonstrated that compared with the control group, incubation under HG significantly enhanced ROS $(\mathrm{P}<0.01$; Fig. $3 \mathrm{~A}$ and $\mathrm{B})$ and MDA $(\mathrm{P}<0.01$; Fig. $3 \mathrm{C})$ production and reduced SOD activity $(\mathrm{P}<0.01$; Fig. 3D). Treatment with RLX significantly suppressed ROS and MDA generation with a concomitant increase in SOD activity (all $\mathrm{P}<0.01$ ) compared with the HG group. Of note, compared with the HG+RLX group, pretreatment with DAPT almost abolished the protective effects of RLX against oxidative stress $(\mathrm{P}<0.05)$.

$R L X$ attenuates $H G$-induced cardiomyocyte hypertrophy and apoptosis via Notch1 signaling. To confirm whether Notch1 signaling is critical for the effects of RLX on HG-induced cardiac hypertrophy and apoptosis, alterations in the expression of Notch1 and its downstream protein, hes1, were examined by RT-qPCR and western blot analysis. As presented in Fig. 4A and B, the expression of Notch1 and hes1 mRNA was significantly decreased in $\mathrm{H} 9 \mathrm{c} 2$ cells exposed to HG compared with that in the control group (all $\mathrm{P}<0.01$ ). Addition of RLX
A
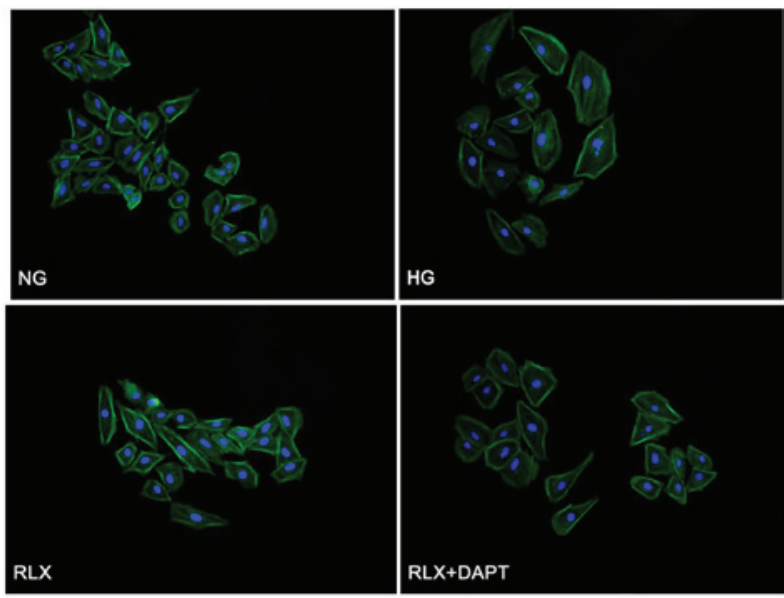

B

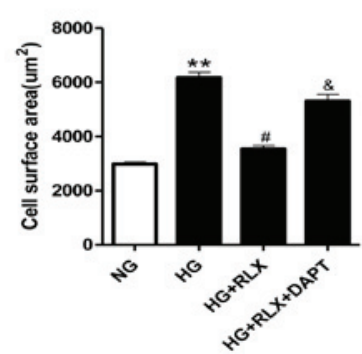

Figure 2. RLX inhibits HG-induced increases in the cell surface area of H9c2 cells. (A) The cell surface area was observed using tetramethylrhodamine B isothiocyanate-labeled phalloidin staining and observation by fluorescence microscopy (magnification, x100; scale bar, $10 \mu \mathrm{m}$ ). Tetramethylrhodamine B isothiocyanate-labeled phalloidin staining was performed to stain the cytoskeleton (green) and DAPI was used to stain the nuclei (blue). (B) Quantitative analysis of the cell surface area. Values are expressed as the mean \pm standard deviation ( $\mathrm{n}=6$ in each group). ${ }^{* *} \mathrm{P}<0.01$ vs. NG group; ${ }^{\text {}} \mathrm{P}<0.05$ vs. HG group; ${ }^{\&} \mathrm{P}<0.05$ vs. HG + RLX group. NG, normal glucose; HG, high glucose; RLX, relaxin.

significantly enhanced the gene expression of Notch1 and hes1 when compared with that in the HG group (all $\mathrm{P}<0.05$ ). Furthermore, pretreatment with DAPT significantly reduced the expression of Notch1 and hes1 mRNA compared with that in the HG+RLX group (all $\mathrm{P}<0.05$ ).

As presented in Fig. 4C and D, the mRNA expression levels of ANP and BNP were increased in the HG group compared with those in the control group (all $\mathrm{P}<0.01$ ). Addition of RLX led to a downregulation of ANP and BNP mRNA expression as compared with that in the HG group (all $\mathrm{P}<0.01$ ). Pretreatment with DAPT led to an upregulation of the mRNA expression of ANP and BNP as compared with that in the HG+RLX group (all $\mathrm{P}<0.01$ ).

These results were further confirmed by western blot analysis (Fig. 5A-D). Overall, it was indicated that RLX attenuated HG-induced cardiomyocyte hypertrophy and apoptosis via mechanisms involving the Notch1 signaling pathway.

Oxidative damage was further assessed through quantitating the protein expression of MnSOD and cytochrome $\mathrm{C}$ by western blot analysis. As presented in Fig. 5E and F, the expression of MnSOD $(\mathrm{P}<0.01)$ in cardiomyocytes exposed to $\mathrm{HG}$ was significantly decreased compared with that in the control group, accompanied with a marked increase in cytochrome $\mathrm{C}(\mathrm{P}<0.01)$. Application of RLX significantly upregulated the expression of MnSOD $(\mathrm{P}<0.05)$ and inhibited cytochrome 
A
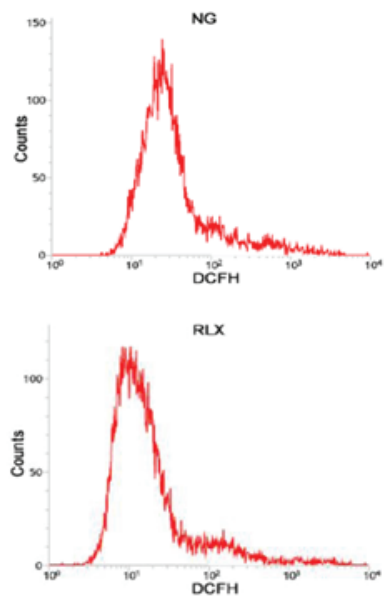

$\mathbf{C}$

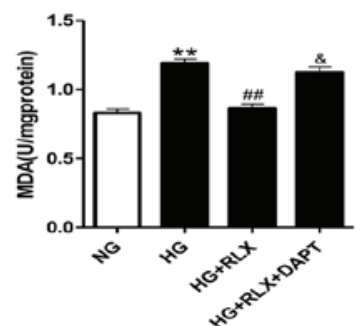

HG
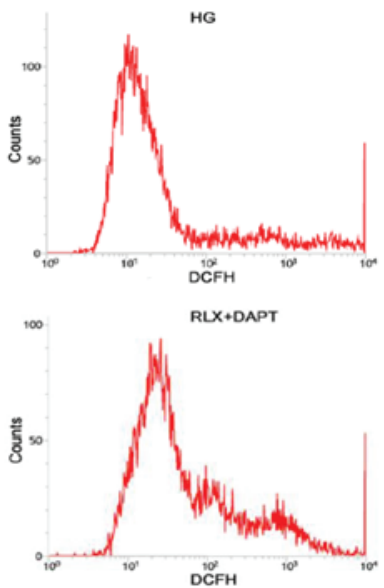

D

B
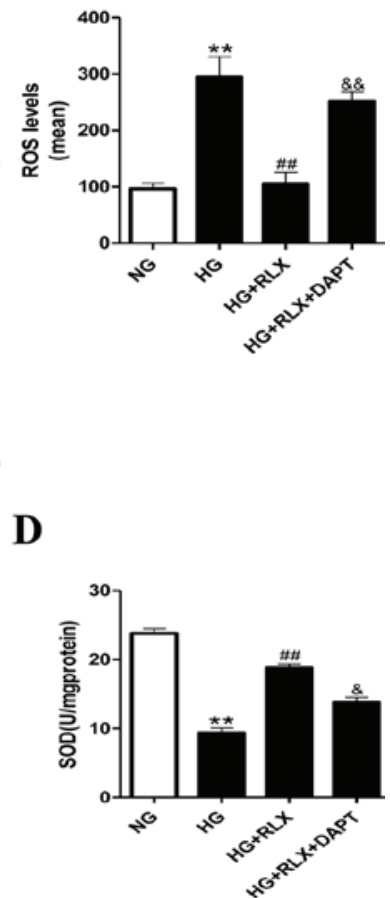

Figure 3. RLX reduces ROS production and MDA formation and increases SOD activity in H9c2 cells exposed to HG. (A) Cellular ROS production was assessed by 2',7'-dichlorofluorescein staining and flow cytometric evaluation. (B) Quantified levels of ROS are displayed in the bar graphs. (C) MDA production and (D) SOD activity were assessed using biochemical assays. Values are expressed as the mean \pm standard deviation ( $\mathrm{n}=6$ in each group). ${ }^{* *} \mathrm{P}<0.01 \mathrm{vs}$. NG group; ${ }^{\# \#} \mathrm{P}<0.01$ vs. HG group; ${ }^{\&} \mathrm{P}<0.05$ and ${ }^{\& \&} \mathrm{P}<0.01 \mathrm{vs.} \mathrm{HG}+\mathrm{RLX}$ group. ROS, reactive oxygen species; SOD, superoxide dismutase; MDA, malondialdehyde; NG, normal glucose; HG, high glucose; RLX, relaxin; DCFH, 2',7'-dichlorofluorescin.

$\mathrm{C}(\mathrm{P}<0.01)$ compared with the HG group. Pretreatment with DAPT significantly abrogated the protective effects of RLX.

Furthermore, the protein levels of caspase-3, which is representative of the extrinsic and intrinsic pathways of apoptosis, were detected by western blot analysis (Fig. 5G). The expression of caspase- 3 was significantly increased in the HG group compared with that in the control group $(\mathrm{P}<0.05)$. However, when compared with that in the HG group, administration of RLX led to a significant downregulation of the expression of caspase-3 $(\mathrm{P}<0.05)$. Pretreatment with DAPT largely abrogated the protective effects of RLX $(\mathrm{P}<0.05)$. These results demonstrated that Notch1 signaling may be involved in the protective role of RLX against cardiac cell apoptosis.

\section{Discussion}

The present study found that incubation under HG decreased cell viability, enhanced oxidative stress and increased cardiomyocyte hypertrophy and caused apoptosis in H9c2 cells. However, RLX treatment increased the cell viability in a doseand time-dependent manner, reduced intracellular oxidative stress, which was concomitant with an inhibition of cardiomyocyte hypertrophy, manifested as increased cell surface area, reduced ANP and BNP content, and decreased apoptosis manifested as increased apoptotic cell death and increased caspase-3 protein expression. Furthermore, RLX treatment activated the expression of Notch1 and hes1 under HG conditions, while the Notch1 inhibitor DAPT largely abolished RLX-induced increases in Notch 1 and hes 1 and prevented
A

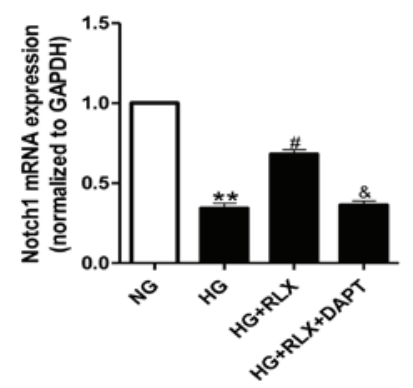

C

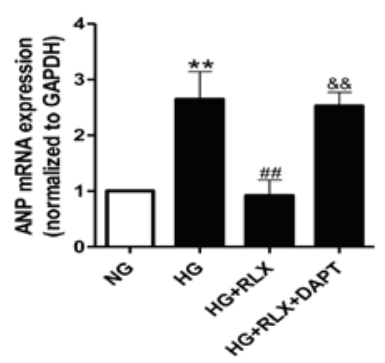

B

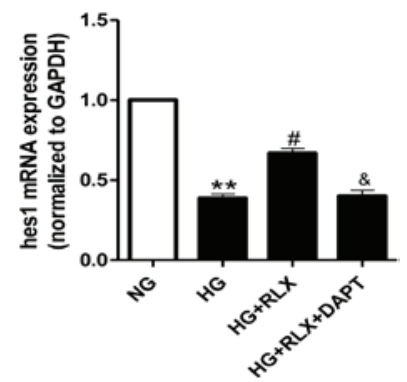

D

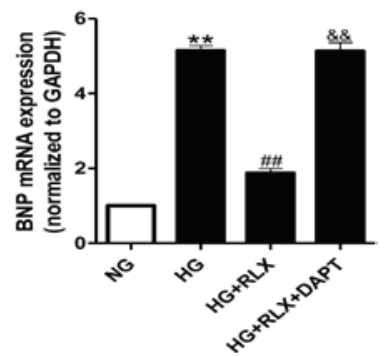

Figure 4. RLX decreases ANP and BNP mRNA levels, and increases Notch1 and hes1 mRNA levels in H9c2 cells exposed to HG. (A) Notch1, (B) hes1, (C) ANP and (D) BNP mRNA levels were quantified by reverse transcription-quantitative polymerase chain reaction. Values are expressed as the mean \pm standard deviation ( $\mathrm{n}=6$ in each group). ${ }^{* *} \mathrm{P}<0.01$ vs. NG group; ${ }^{\#} \mathrm{P}<0.05$ and ${ }^{\# \#} \mathrm{P}<0.01$ vs. HG group; ${ }^{\circledR} \mathrm{P}<0.05$ and ${ }^{\& \&} \mathrm{P}<0.01$ vs. $\mathrm{HG}+\mathrm{RLX}$ group. NG, normal glucose; HG, high glucose; RLX, relaxin; ANP, atrial natriuretic polypeptide; BNP, brain natriuretic polypeptide; hes1, hairy and enhancer of split 1 . 


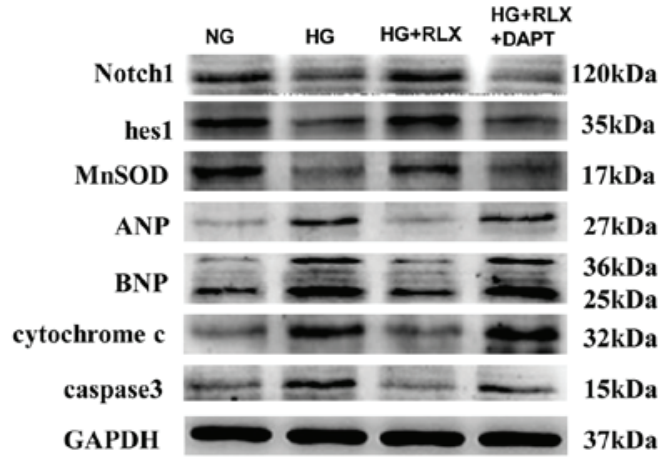

B

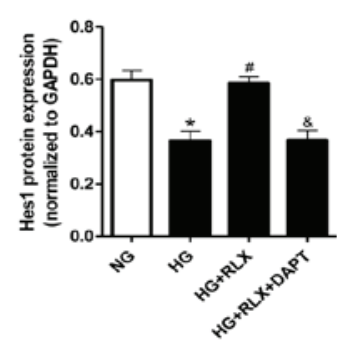

C

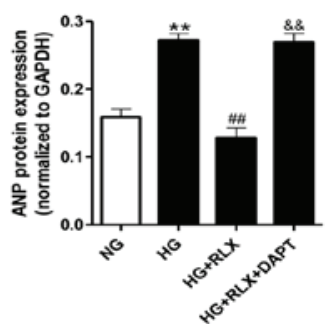

$\mathbf{F}$

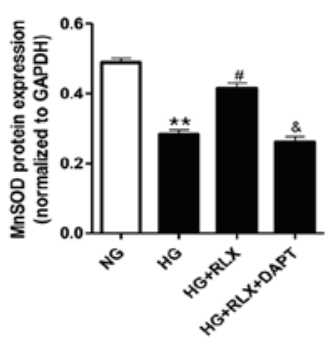

D

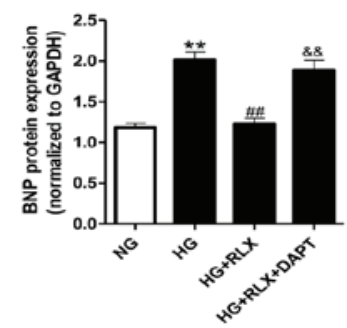

A

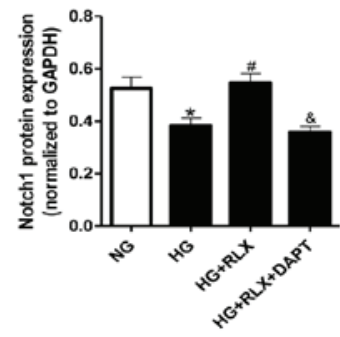

G

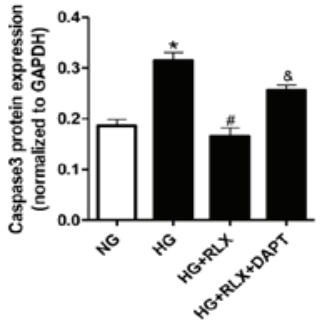

Figure 5. RLX decreases ANP, BNP, caspase-3 and cytochrome C protein levels, and increases MnSOD activity, as well as activator of Notch1 and hes1 protein expression in H9c2 cells exposed to HG. Protein expression was measured by western blot analysis. (A-G) Quantitative analysis of (A) Notch1, (B) hes1, (C) ANP and (D) BNP, (E) MnSOD, (F) cytochrome C and (G) caspase 3. For the quantitative evaluation of BNP, the two bands obtained were for two different glycosylated BNP precursors and both were taken into account. Values are expressed as the mean \pm standard deviation ( $\mathrm{n}=6$ in each group). ${ }^{*} \mathrm{P}<0.05$ and ${ }^{* *} \mathrm{P}<0.01$ vs. NG group; ${ }^{*} \mathrm{P}<0.05$ and ${ }^{\# \#} \mathrm{P}<0.01$ vs. HG group; ${ }^{\&} \mathrm{P}<0.05$ and ${ }^{~ \& \&} \mathrm{P}<0.01$ vs. HG + RLX group. NG, normal glucose; HG, high glucose; RLX, relaxin; ANP, atrial natriuretic polypeptide; BNP, brain natriuretic polypeptide; hes1, hairy and enhancer of split 1; MnSOD, manganese superoxide dismutase.

RLX-mediated cardiomyocyte protection, suggesting that RLX has a role in cardioprotection at least in part through the Notch1/hes1 signaling pathway.

RLX, an insulin-like peptide hormone, does not mimic the metabolic effects of insulin, but increased RLX levels were demonstrated to improve insulin sensitivity in diabetic patients (24). Previous studies have indicated that RLX levels were significantly lower in patients with diabetes, whereas a compensatory increase in RLX levels helped pregnant women with diabetes mellitus overcome increased insulin resistance $(25,26)$. In fact, clinical and experimental studies have suggested that RLX protects the heart from $\mathrm{HG}$-induced myocardial damage, possibly through its vasodilative, antiangiogenic (7), antifibrotic (13) and antiapoptotic actions (14). Although the effects of RLX on HG-induced cardiomyocyte apoptosis were previously investigated, the molecular mechanism has remained to be determined. In addition, the effect of RLX on the interaction between hyperglycemia and hypertrophy, as well as the underlying intracellular signaling pathways have remained elusive.

Increased oxidative stress is closely associated with cardiac hypertrophy and apoptosis, which has been reported in numerous studies $(5,27)$. Evidence from animal and cell culture experiments indicated that ROS elimination inhibited hyperglycemia-induced hypertrophy and apoptosis (4). Several studies have demonstrated that RLX is a potent antioxidant effector that protects against the development of certain diseases by reducing ROS production and maintaining nitric oxide availability $(28,29)$. In addition, studies have revealed that RLX prevents HG- (14) or ischemia/reperfusion-induced (11) myocardial apoptosis in neonatal rat ventricular myocytes by reducing oxidative damage and inhibiting apoptosis-associated pathways. The present findings are in accordance with a previous study indicating that RLX treatment inhibited HG-induced cardiomyocyte apoptosis, manifested as an increased apoptotic cell index, and upregulated the protein synthesis of caspase-3, as a key mediator of apoptosis (30).

Previous studies indicated that endogenous RLX is upregulated in the hypertrophic heart and that RLX treatment reversed hypertrophy in spontaneously hypertensive rats; RLX treatment was also demonstrated to counteract phenylephrine-induced cardiomyocyte hypertrophy by inhibiting activated extracellular signal-regulated kinase (ERK)-1/2 (15,16). However, Moore et al (16) reported that RLX alone failed to suppress phenylephrine-induced 
cardiomyocyte hypertrophy, whereas RLX indirectly inhibited cardiac fibroblast-conditioned medium-induced hypertrophy and directly inhibited $\mathrm{H}_{2} \mathrm{O}_{2}$-induced apoptosis through activation of the Akt and ERK pathways. In addition, Xu et al (31) demonstrated that endogenous RLX had no significant effect on chronic pressure overload-induced cardiac hypertrophy and fibrosis. Therefore, the effect of RLX on cardiac hypertrophy is currently controversial. The present study reported that administration of RLX inhibited $\mathrm{HG}$-induced increases in cell surface area and the expression of ANP and BNP as markers of hypertrophy at the mRNA and protein level. To our knowledge, our study has revealed, for the first time, RLX inhibited HG-induced cardiomyocyte hypertrophy.

In order to verify whether the prevention of HG-induced hypertrophy and apoptosis by RLX was through inhibition of oxidative stress, the ROS-sensitive DCFH-DA dye was used to measure intracellular ROS levels. MDA as a byproduct of lipid peroxidation and SOD as an antioxidant enzyme were assessed as typical biomarkers of oxidative insults. In addition, MnSOD (a mitochondrial antioxidant enzyme) and cytochrome C (32) are important members of the intrinsic mitochondrial pathway. The mitochondria are a major source of ROS. The present study indicated that RLX treatment reduced the intracellular ROS and MDA levels, enhanced SOD activity, and increased the protein synthesis of MnSOD, as well as decreased the protein expression of cytochrome $\mathrm{C}$, which was concomitant with the inhibition of cardiomyocyte hypertrophy and apoptosis in H9c2 cells under HG treatment. Therefore, the present results collectively demonstrated that RLX attenuated HG-induced cardiomyocyte hypertrophy and apoptosis via oxidative stress.

Previous studies have indicated that the activation of Notch1 signaling improves diabetic wound healing (33) and diabetes-induced microvasculopathy (34) in a streptozotocin-induced mouse model of diabetes mellitus. Another study also demonstrated that activation of the Notch1 and phosphoinositide-3 kinase/Akt signaling pathways is critical for the prevention of DCM by inhibiting HG-induced cardiomyocyte apoptosis (35). Basu et al (36) indicated that hyperglycemia inhibited the expression of Notch1 and its downstream effectors in chick embryos, as well as $\mathrm{H} 9 \mathrm{c} 2$ and endocardial-derived cells, and increased the risk of congenital heart defects in maternal mice with diabetes mellitus. In addition, animal studies have revealed that Notch1 overexpression protects against myocardial ischemia-reperfusion and ischemic preconditioning, as well as ischemic postconditioning injury by reducing cardiomyocyte apoptosis, which involves reduction of oxidative/nitrative stress $(37,38)$. The involvement of Notch1 in cardiac hypertrophy has been reported previously. For instance, the expression of Notch1 and hes1 is downregulated in the one-kidney one clip mouse model (39) and in the adult heart under pressure overload-induced cardiac hypertrophy (19). Conversely, Notch inhibition accelerates the development of cardiac hypertrophy and fibrosis. Therefore, the aforementioned studies collectively indicated that Notch1 signaling has a pivotal role in cardiac hypertrophy and apoptosis associated with various cardiac diseases.

Boccalini et al (11) and Zhou et al (22) reported that the protective effects of RLX may depend on the mechanism of
Notch1 activation. Their studies emphasized the antiapoptotic and antifibrotic actions of RLX using a model of hypoxia/reoxygenation injury or a model of cardiac fibrosis. However, the focus of the present study was to investigate the effect of RLX on HG-induced cardiomyocyte hypertrophy and apoptosis using a model of hyperglycemia-induced injury in vitro, and to assess the possible molecular mechanism, which was different from the studies by Boccalini et al (11) and Zhou et al (22). In addition, Boccalini et al (11) demonstrated that RLX inhibits hypoxia/reoxygenation-induced apoptosis in $\mathrm{H} 9 \mathrm{c} 2$ rat cardiomyoblasts. While RLX was previously reported to regulate HG-induced cardiac apoptosis, it had remained elusive whether the underlying mechanism involves the Notch1 signaling pathway. Of note, the present study was the first, to the best of our knowledge, to reveal that RLX inhibits HG-induced cardiomyocyte apoptosis through activation of Notch1 signaling.

To investigate the Notch1 pathway and whether it has a pivotal role in the effects of RLX on HG-induced cardiac hypertrophy and apoptosis, the highly active $\gamma$-secretase inhibitor DAPT was used to block the Notch1 pathway. The results indicated that HG decreased the expression of Notch1 and hes1, and blocking the Notch1 pathway with DAPT increased cardiac hypertrophy and apoptosis. It was revealed that DAPT largely, but not fully offset the cytoprotective effects of RLX, suggesting that multiple signaling pathways may be activated by RLX in cardiac myocytes. Therefore, these results collectively indicated that RLX-mediated inhibition of HG-induced cardiac hypertrophy and apoptosis is at least partly due to activation of the Notch1 signaling pathway.

However, the present study had several limitations. First, all experiments were performed using the H9c2 cell line, and further exploration should be done using primary neonatal cardiomyocytes or in vivo experiments. Furthermore, the expression of ANP and BNP was examined in the present study, while these proteins do not adequately represent hypertrophy in vivo. Therefore, more specific markers for the hypertrophic heart should be considered in the future. Additional studies are required to clarify whether the anti-hypertrophic and anti-apoptotic action of RLX is mediated via multiple signaling pathways, and to investigate how the Notch1 pathway is interlinked and cooperates to mediate the effects of RLX. Further studies are required to address these questions.

In conclusion, the results of the present study demonstrated that RLX protects H9c2 cells from HG-induced hypertrophy and apoptosis at least partly through the activation of Notch1 signaling, and may be associated with the inhibition of oxidative stress. The present findings provided novel insight into the molecular mechanisms of RLX-mediated cardioprotection as well as further evidence for selecting RLX as a novel therapeutic drug for the treatment of DCM.

\section{Acknowledgements}

This study was supported by the National Natural Science Foundation of China (grant no. 81300140).

\section{References}

1. Chavali V, Tyagi SC and Mishra PK: Predictors and prevention of diabetic cardiomyopathy. Diabetes Metab Syndr Obes 6: 151-160, 2013. 
2. Rubler S, Dlugash J, Yuceoglu YZ, Kumral T, Branwood AW and Grishman A: New type of cardiomyopathy associated with diabetic glomerulosclerosis. Am J Cardiol 30: 595-602, 1972.

3. Hayat SA, Patel B, Khattar RS and Malik RA: Diabetic cardiomyopathy: Mechanisms, diagnosis, and treatment. Clin Sci (Lond) 107: 539-557, 2004.

4. Zhou $X$ and $\mathrm{Lu} \mathrm{X}$ : The role of oxidative stress in high glucose-induced apoptosis in neonatal rat cardiomyocytes. Exp Biol Med (Maywood) 238: 898-902, 2013.

5. Huynh K, Bernardo BC, McMullen JR and Ritchie RH: Diabetic cardiomyopathy: Mechanisms and new treatment strategies targeting antioxidant signaling pathways. Pharmacol Ther 142: 375-415, 2014.

6. Sherwood OD: Relaxin's physiological roles and other diverse actions. Endocr Rev 25: 205-234, 2004.

7. Bitto A, Irrera N, Minutoli L, Calò M, Lo Cascio P, Caccia P, Pizzino G, Pallio G, Micali A, Vaccaro M, et al: Relaxin improves multiple markers of wound healing and ameliorates the disturbed healing pattern of genetically diabetic mice. Clin Sci 125: 575-585, 2013.

8. Samuel CS, Unemori EN, Mookerjee I, Bathgate RA, Layfield SL Mak J, Tregear GW and Du XJ: Relaxin modulates cardiac fibroblast proliferation, differentiation, and collagen production and reverses cardiac fibrosis in vivo. Endocrinology 145: 4125-4133, 2004.

9. Taylor MJ and Clark CL: Evidence for a novel source of relaxin: Atrial cardiocytes. J Endocrinol 143: R5-R8, 1994.

10. Formigli L, Francini F, Nistri S, Margheri M, Luciani G, Naro F Silvertown JD, Orlandini SZ, Meacci E and Bani D: Skeletal myoblasts overexpressing relaxin improve differentiation and communication of primary murine cardiomyocyte cell cultures. J Mol Cell Cardiol 47: 335-345, 2009.

11. Boccalini G, Sassoli C, Formigli L, Bani D and Nistri S: Relaxin protects cardiac muscle cells from hypoxia/reoxygenation injury: Involvement of the Notch-1 pathway. FASEB J 29: 239-249, 2015

12. Tan YY, Wade JD, Tregear GW and Summers RJ: Quantitative autoradiographic studies of relaxin binding in rat atria, uterus and cerebral cortex: Characterization and effects of oestrogen treatment. Br J Pharmacol 127: 91-98, 1999.

13. Wang P, Li HW, Wang YP, Chen $\mathrm{H}$ and Zhang P: Effects of recombinant human relaxin upon proliferation of cardiac fibroblast and synthesis of collagen under high glucose condition. J Endocrinol Invest 32: 242-247, 2009.

14. Zhang X, Ma X, Zhao M, Zhang B, Chi J, Liu W, Chen W, Fu Y, Liu Y and Yin X: $\mathrm{H} 2$ and $\mathrm{H} 3$ relaxin inhibit high glucose-induced apoptosis in neonatal rat ventricular myocytes. Biochimie 108: 59-67, 2015.

15. Parikh A, Patel D, McTiernan CF, Xiang W, Haney J, Yang L, Lin B, Kaplan AD, Bett GC, Rasmusson RL, et al: Relaxin suppresses atrial fibrillation by reversing fibrosis and myocyte hypertrophy and increasing conduction velocity and sodium current in spontaneously hypertensive rat hearts. Circ Res 113: 313-321, 2013

16. Moore XL, Tan SL, Lo CY, Fang L, Su YD, Gao XM, Woodcock EA, Summers RJ, Tregear GW, Bathgate RA and Du XJ: Relaxin antagonizes hypertrophy and apoptosis in neonatal rat cardiomyocytes. Endocrinology 148: 1582-1589, 2007.

17. High FA and Epstein JA: The multifaceted role of Notch in cardiac development and disease. Nat Rev Genet 9: 49-61, 2008.

18. Dabral S, Tian X, Kojonazarov B, Savai R, Ghofrani HA Weissmann N, Florio M, Sun J, Jonigk D, Maegel L, et al: Notch1 signalling regulates endothelial proliferation and apoptosis in pulmonary arterial hypertension. Eur Respir J 48: 1137-1149, 2016.

19. Nemir M, Metrich M, Plaisance I, Lepore M, Cruchet S, Berthonneche C, Sarre A, Radtke F and Pedrazzini T: The Notch pathway controls fibrotic and regenerative repair in the adult heart. Eur Heart J 35: 2174-2185, 2014.

20. Urbanek K, Cabral-da-Silva MC, Ide-Iwata N, Maestroni S, Delucchi F, Zheng H, Ferreira-Martins J, Ogórek B, D'Amario D, Bauer M, et al: Inhibition of notch1-dependent cardiomyogenesis leads to a dilated myopathy in the neonatal heart. Circ Res 107: 429-441, 2010

21. Sassoli C, Chellini F, Pini A, Tani A, Nistri S, Nosi D, Zecchi-Orlandini S, Bani D and Formigli L: Relaxin prevents cardiac fibroblast-myofibroblast transition via notch-1-mediated inhibition of TGF- $\beta /$ Smad3 signaling. PLoS One 8: e63896, 2013.
22. Zhou X, Chen X, Cai JJ, Chen LZ, Gong YS, Wang LX, Gao Z, Zhang HQ, Huang WJ and Zhou H: Relaxin inhibits cardiac fibrosis and endothelial-mesenchymal transition via the Notch pathway. Drug Des Devel Ther 9: 4599-4611, 2015.

23. Livak KJ and Schmittgen TD: Analysis of relative gene expression data using real-time quantitative PCR and the 2(-Delta Delta C(T)) method. Methods 25: 402-408, 2001

24. Bani D, Pini A and Yue SK: Relaxin, insulin and diabetes: An intriguing connection. Curr Diabetes Rev 8: 329-335, 2012

25. Zhang X, Zhu M, Zhao M, Chen W, Fu Y, Liu Y, Liu W, Zhang B, Yin X and Bai B: The plasma levels of relaxin-2 and relaxin-3 in patients with diabetes. Clin Biochem 46: 1713-1716, 2013.

26. Whittaker PG, Edwards JR, Randolph C, Büllesbach EE, Schwabe C and Steinetz BG: Abnormal relaxin secretion during pregnancy in women with type 1 diabetes. Exp Biol Med (Maywood) 228: 33-40, 2003.

27. Gupta MK, Neelakantan TV, Sanghamitra M, Tyagi RK, Dinda A, Maulik S, Mukhopadhyay CK and Goswami SK: An assessment of the role of reactive oxygen species and redox signaling in norepinephrine-induced apoptosis and hypertrophy of H9c2 cardiac myoblasts. Antioxid Redox Signal 8: 1081-1093, 2006.

28. Ng HH, Jelinic M, Parry LJ and Leo CH: Increased superoxide production and altered nitric oxide-mediated relaxation in the aorta of young but not old male relaxin-deficient mice. Am J Physiol Heart Circ Physiol 309: H285-H296, 2015.

29. Willcox JM and Summerlee AJ: Relaxin protects astrocytes from hypoxia in vitro. PLoS One 9: e90864, 2014.

30. Lakhani SA, Masud A, Kuida K, Porter GA Jr, Booth CJ, Mehal WZ, Inayat I and Flavell RA: Caspases 3 and 7: Key mediators of mitochondrial events of apoptosis. Science 311: 847-851, 2006.

31. Xu Q, Lekgabe ED, Gao XM, Ming Z, Tregear GW, Dart AM, Bathgate RA, Samuel CS and Du XJ: Endogenous relaxin does not affect chronic pressure overload-induced cardiac hypertrophy and fibrosis. Endocrinology 149: 476-482, 2008.

32. Cai L, Li W, Wang G, Guo L, Jiang Y and Kang YJ: Hyperglycemia-induced apoptosis in mouse myocardium: mitochondrial cytochrome C-mediated caspase-3 activation pathway. Diabetes 51: 1938-1948, 2002.

33. Yang RH, Qi SH, Shu B, Ruan SB, Lin ZP, Lin Y, Shen R, Zhang FG, Chen XD and Xie JL: Epidermal stem cells (ESCs) accelerate diabetic wound healing via the Notch signalling pathway. Biosci Rep 36: pii: e00364, 2016.

34. Yoon CH, Choi YE, Cha YR, Koh SJ, Choi JI, Kim TW, Woo SJ, Park YB, Chae IH and Kim HS: Diabetes-induced jagged1 overexpression in endothelial cells causes retinal capillary regression in a murine model of diabetes mellitus: Insights into diabetic retinopathy. Circulation 134: 233-247, 2016

35. Zhang J, Li B, Zheng Z, Kang T, Zeng M, Liu Y and Xia B: Protective effects of Notch1 signaling activation against high glucose-induced myocardial cell injury: Analysis of its mechanisms of action. Int J Mol Med 36: 897-903, 2015.

36. Basu M, Bosse K and Garg V: Notch1 Haploinsufficiency Increases Risk of Congenital Heart Defects in the Setting of Maternal Diabetes by an Epigenetic Mechanism. Circulation 130:19285, 2014.

37. Pei H, Yu Q, Xue Q, Guo Y, Sun L, Hong Z, Han H, Gao E, $\mathrm{Qu}$ Y and Tao L: Notch1 cardioprotection in myocardial ischemia/reperfusion involves reduction of oxidative/nitrative stress. Basic Res Cardiol 108: 373, 2013.

38. Zhou XL, Wan L, Xu QR, Zhao Y and Liu JC: Notch signaling activation contributes to cardioprotection provided by ischemic preconditioning and postconditioning. J Transl Med 11: 251, 2013.

39. Nemir M, Jordan N, Croquelois A, Domenighetti A and Pedrazzini T: Notch signaling: A potential regulator of cardiac response to hypertrophic stimuli. J Mol Cell Cardiol 42 (Suppl): S134-S135, 2007.

This work is licensed under a Creative Commons Attribution-NonCommercial-NoDerivatives 4.0 International (CC BY-NC-ND 4.0) License. 